\title{
Robustness of Estimators of the Squared Multiple Correlation and Squared Cross-Validity Coefficient to Violations of Multivariate Normality
}

\author{
Fritz Drasgow \\ Yale University \\ Vell d. Dorans \\ Educational Testing Service
}

\begin{abstract}
A monte carlo experiment was conducted to evaluate the robustness of two estimators of the population squared multiple correlation $\left(R_{p}^{2}\right)$ and one estimator of the population squared cross-validity coefficient $\left(R_{c v}^{2}\right)$ to a common violation of multivariate normality. Previous research has shown that these estimators are approximately unbiased when independent and dependent variables follow a joint multivariate normal distribution. The particular violation of multivariate normality studied here consisted of a dependent variable that may assume only a few discrete values. The discrete dependent variable was simulated by categorizing an underlying continuous variable that did satisfy the multi-
\end{abstract}

\begin{abstract}
variate normality condition. Results illustrate the attenuating effects of categorization upon $R_{p}^{2}$ and $R_{c v}^{2}$. In addition, the distributions of sample squared multiple correlations and sample squared cross-validity coefficients are affected by categorization mainly through the attenuations of $\mathbb{R}_{p}^{2}$ and $\mathbb{R}_{c v}^{2}$ Consequently, the formula estimators of $R_{p}^{2}$ and $R_{c r}^{2}$ were found to be as accurate and unbiased with discrete dependent variables as they were with continuous dependent variables. Substantive researchers who use categorical dependent variables, perhaps obtained by rating scale judgments, can justifiably employ any of the three estimators examined here.
\end{abstract}

Multiple regression is a method used in many areas of psychology to study the relation between a set of independent variables and a dependent variable. There seem to be two distinct functions served by these studies. In research investigating theoretical relations, interest is focused on the population regression equation. Applied research efforts, in contrast, are usually concerned with the long run effectiveness of a particular sample regression equation. In neither case is the effectiveness of a sample regression equation in its derivation sample of primary interest.

The null hypothesis that all regression weights are zero in the population can be tested by a wellknown procedure. Researchers frequently wish to go beyond this significance test and consider the magnitude of relation between the set of independent variables and the dependent variable. Unfortunately, the sample squared multiple correlation, $\mathbb{R}_{s}^{2}$, can provide misleading information about the magnitude of the relationship, particularly if sample size, $N$, is not substantially larger than the number of independent variables, $k$.

To eliminate the bias of $R_{s}^{2}$ a number of "shrinkage" formulas have been developed. Considered here are (1) two formulas for estimating the squared multiple correlation, $R_{p}^{2}$, which results when the

APPLIED PSYCHOLOGICAL MEASUREMENT

Vol. 6. No. 2, Spring 1982, pp. 185-200

(c) Copyright 1982 Applied Psychological Measurement Inc.

0146-6216/82/020185-16\$1.80 
population multiple regression equation is applied in the population and (2) a formula for estimating the squared cross-validity coefficient, $\mathbb{R}_{c v}^{2}$, which results when a sample multiple regression equation is applied in the population.

The first estimator of $R_{p}^{2}$, usually attributed to Wherry (1931), is

$$
\hat{\mathrm{R}}_{\mathrm{p}}^{2}=1-\frac{\mathrm{N}-1}{\mathrm{~N}-\mathrm{k}-1}\left(1-\mathrm{R}_{\mathrm{s}}^{2}\right) \text {. }
$$

This equation is appropriate for the "fixed $X$ " or regression model (i.e., values of the independent variables are fixed or predetermined by the investigator) when an intercept parameter is included in the regression equation. The equation actually presented by Wherry (1931) is

$$
\hat{R}_{p}^{2}=1-\frac{N-1}{N-k}\left(1-R_{S}^{2}\right)
$$

which can be derived when there is no intercept parameter. Because virtually all psychologists include a constant in their regression equations, Equation 2 is not considered further. Note, however, that Equation 2 does occasionally appear in the literature (see, for example, Nunnally, 1978, p. 180).

The second formula for estimating $R_{p}^{2}$ examined here is the three-term expansion (Herzberg, 1969) of the Olkin-Pratt (1958) estimator,

$$
\hat{\mathrm{R}}_{\mathrm{p}}{ }^{2}=1-\frac{\mathrm{N}-3}{\mathrm{~N}-\mathrm{k}-1}\left(1-\mathrm{R}_{\mathrm{s}}{ }^{2}\right)-\frac{\mathrm{N}-3}{\mathrm{~N}-\mathrm{k}-1} \frac{2}{\mathrm{~N}-\mathrm{k}+1}\left(1-\mathrm{R}_{\mathrm{s}}{ }^{2}\right)^{2} \text {. }
$$

This equation is appropriate under the conditions of the correlation or "random $X$ " model (i.e., independent and dependent variables follow a multivariate normal distribution).

The precisions of Equations 1 and 3 can be evaluated (at least approximately) by analytic methods when the assumptions underlying the equations are met. For example, the Olkin-Pratt (1958) estimator is unbiased and the three-term expansion in Equation 3 of the infinite series in the Olkin-Pratt estimator is approximately unbiased.

Browne (1975) developed an estimator of $R_{c v}^{2}$ that is appropriate for the random $X$ model. It is

$$
\hat{R}_{C V}{ }^{2}=\frac{(N-k-3) \hat{R}_{p}^{4}-\hat{R}_{p}^{2}}{(N-2 k-2) \hat{R}_{p}{ }^{2}+k} .
$$

Browne suggested estimating $R_{p}^{2}$ via Equation 1 and using

$$
\hat{R}_{p}^{4}=\left(\hat{R}_{p}^{2}\right)^{2}-\frac{2 k\left(1-\hat{R}_{p}^{2}\right)^{2}}{(N-1)(N-k+1)}
$$

to estimate $R_{p}^{4}$.

In a recent monte carlo study, Drasgow, Dorans, and Tucker (1979) found that Equation 4 performed quite well within the scope of their simulation. It is important to note that the conditions simulated by Drasgow et al. (1979) precisely matched the assumptions of the random $X$ model.

In sum, when used under appropriate conditions, Equations 1,3, and 4 all appear approximately unbiased. In addition, the standard errors for Equations 1 and 3 may be approximated by analytic methods using relations presented by Kendall and Stuart (1979, pp. 362-363), and the research of Drasgow et al. (1979) can be used to examine the standard error of Equation 4.

The present research examines the effects of violating an important assumption that underlies Equations 1, 3, and 4. First studied are the effects of the violation on the sampling distributions of $\mathbb{R}_{s}^{2}$ 
and $R_{c v}^{2}$ Clearly, if these distributions are affected in unpredictable ways, it is unlikely that Equations 1,3 , and 4 will be effective.

\section{A Discrete Dependent Variable}

The violation of multivariate normality considered here is one that occurs frequently in psychology. In particular, the dependent variable is not assumed to be continuous but, instead, is discrete. Three examples illustrate the categorical nature of important dependent variables in psychology.

The first example comes from personnel psychology. Ability tests are commonly used to predict job performance. The tests may assess cognitive, clerical, or physical abilities. It is unfortunate, but common, to see job performance assessed by a 7-point scale that asks the worker's supervisor to rate "overall job performance."

Categorical dependent variables are also encountered in research investigating consequences of workers' affective responses to their work and work role in the organization. Probably the most widely used instrument for measuring various facets of job satisfaction is Smith, Kendall, and Hulin's (1969) Job Descriptive Index (JDI). Scores on JDI scales are used to predict categorical variables such as turnover (which is dichotomous), absenteeism, and grievances filed.

Educational measurement provides a third example of a categorical dependent variable. Firstyear graduate grade-point average is a dependent variable frequently used in studies that investigate the validity of standardized aptitude tests such as the Graduate Record Examination. For six courses that are graded on a common 4-point scale (e.g., 1, 2, 3, and 4), the grade-point average scale contains at most 19 unique scale values, ranging from 1.0 to 4.0 in intervals of .167. In practice, however, it is common to observe severe restriction of range. If only grades of "A" and " $\mathrm{B}$ " are assigned to students, a dependent variable having seven or less scale values will result.

In all three of the examples described, the independent variables, although discrete, have a large number of categories. Frequency distributions are typically unimodal with gradually decreasing tails. The assumption that the independent variables follow a multivariate normal distribution probably does not do gross violence to the data. The dependent variables, in contrast, do not appear remotely normal. Only a limited number of response categories are available, and there may be restriction of range within these few categories. Consequently, the violation of multivariate normality investigated here consists of the failure of the dependent variable to have a marginal normal distribution and be jointly normal with the independent variables.

\section{Psychonetrie Model}

Although the dependent variables under consideration are not normally distributed, it appears reasonable to assume that there is a latent variable underlying the observed dependent variable that meets the multivariate normality assumption. The categorical dependent variable $Y$ is therefore assumed to be formed by imposing a set of thresholds $t_{j}$ or category boundaries on a continuous latent variable $Y$ :

$$
Y=\left\{\begin{array}{l}
1 \text { if } Y<t_{1} \\
j \text { if } t_{j-1} \leq Y<t_{j}, j=2, \ldots g,-1 \\
g \text { if } Y \geq t_{g-1} .
\end{array}\right.
$$


The independent variables and $Y$ are assumed to follow a joint multivariate normal distribution. Without loss of generality, it can be assumed that $Y$ has mean zero and unit variance.

\section{Effects of Polychotonnizing Y}

A consequence of the assumption that $Y$ and the independent variables follow a multivariate normal distribution is that $Y$ and any individual independent variable, $X_{i}$, will follow a bivariate normal distribution. Let $r_{i}$ denote the correlation between $Y$ and $X_{i}$. Olsson, Drasgow, and Dorans (1982) have shown that if the discrete variable $Y$ is formed from $Y$ through Equation 6 , then the correlation between $X_{i}$ and $Y$ is

$$
\underline{r}_{d}=r\left(X_{i}, Y\right)=q r_{i} \text {. }
$$

In Equation $7 q$ is

$$
q=\frac{1}{\sigma_{y}} \sum_{j=1}^{g-1} \phi\left(t_{j}\right),
$$

where $\sigma_{y}$ is the standard deviation of $Y$, and $\phi\left(t_{j}\right)$ is the height of the normal curve at $t_{j}$ :

$$
\phi\left(t_{j}\right)=\frac{1}{\sqrt{2 \pi}} \exp \left\{-\frac{t_{j}^{2}}{2}\right\} \text {. }
$$

A more general form of Equation 8 can be derived that does not constrain the scale values of the categorical criterion to be successive integers, as in Equation 6(Olsson et al., 1982). However, the special case of successive integer scoring is studied here because most researchers use consecutive integers or some linear transformation of consecutive integers.

The scalar $q$ is called the categorization attemation factor because it provides a precise measure of the extent to which categorization reduces the linear relation between $X_{i}$ and $Y$. It is apparent that $q$ depends upon the number and location of the thresholds $t_{j}$. Thus, categorization attenuates $r$ in the way that would be expected.

As implied by its name, the polyserial correlation is a generalization of the biserial correlation. When $Y$ is dichotomized, it is possible to rewrite Equation 7 in the form of the relation between point biserial and biserial correlations presented by $\mathbb{L}$ ord and Novick (1968, p. 340). Lord and Novick illustrate the effects of dichotomizing $Y$ by presenting a table showing $q$ for several thresholds. For example, the maximum value of $q$ is .798 , which occurs when $Y$ is dichotomized at its median.

Equations 7 and 8 can also be used to examine the effects of polychotomizing $Y$ in a particular manner. Table 1 contains the eight sets of thresholds used in this study. The term "threshold vector" is used to denote a set of thresholds for categorizing $\gamma$. The values of $q$ associated with the threshold vectors in Table 1 range from .976 to .637 , and the number $g$ of categories ranges from 2 to 11 .

There are a number of interesting points that can be made from examining Table 1. First, note that with only seven well-spaced categories $r_{d}$ can reach $97.6 \%$ of $r_{i}$. In other analyses (not presented here), values of $q$ were found to be $.898,939, .960$, and .997 for $g$ equal to $3,4,5$, and 25 , respectively. However, simple expressions for the values of $t_{j}$ that maximize $q$ for a given $g$ have not been derived. A simple procedure that yields large values of $q$ involves placing the $t_{j}$ symmetrically about 0 (i.e., the mean of $Y$ and spaced such that the probabilities of categories approximate the normal density function. 


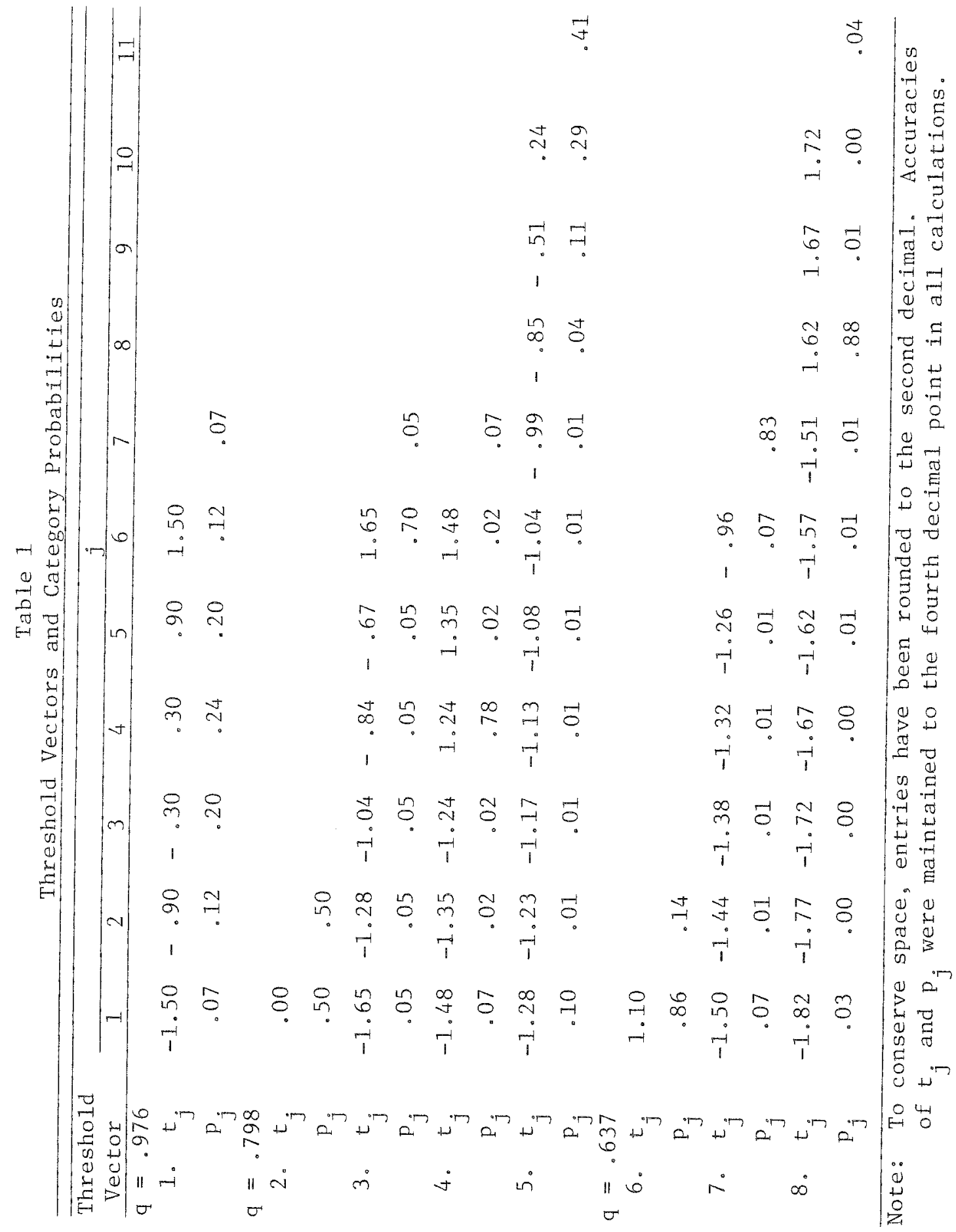

Downloaded from the Digital Conservancy at the University of Minnesota, http://purl.umn.edu/93227.

May be reproduced with no cost by students and faculty for academic use. Non-academic reproduction requires payment of royalties through the Copyright Clearance Center, http://www.copyright.com/ 
A second conclusion evident from Table 1 is that there is not a unique threshold vector for each value of $q$. Instead, many threshold vectors can be selected to produce the same $q$. Table 1 also shows that threshold vectors with differing values of $g$ can yield identical values of $q$.

One further observation concerning Table 1 is that increasing $g$ does not necessarily increase $q$. This point is particularly important to researchers who must decide how many categories to use for their rating scales. Note that the second threshold vector, which produces a dichotomous $Y$, has a larger value of $q$ than the eighth threshold vector, which yields 11 categories of $Y$. Table 1 illustrates that the location of the thresholds is very important in determining $q$.

Table 2 presents the skewness and kurtosis of the eight discrete variables that result from the threshold vectors in Table 1. These moment ratios are also presented for the normal, uniform, and $\chi^{2}$ (one degree of freedom) distributions. The skewness of the discrete variables ranges from symmetry to about the skew of the $\chi^{2}$ distribution. Kurtosis values range from flatter than the uniform distribution to almost as peaked as the $\chi^{2}$ distribution. It is clear that many of these moment ratios depart substantially from the values for the nomal distribution.

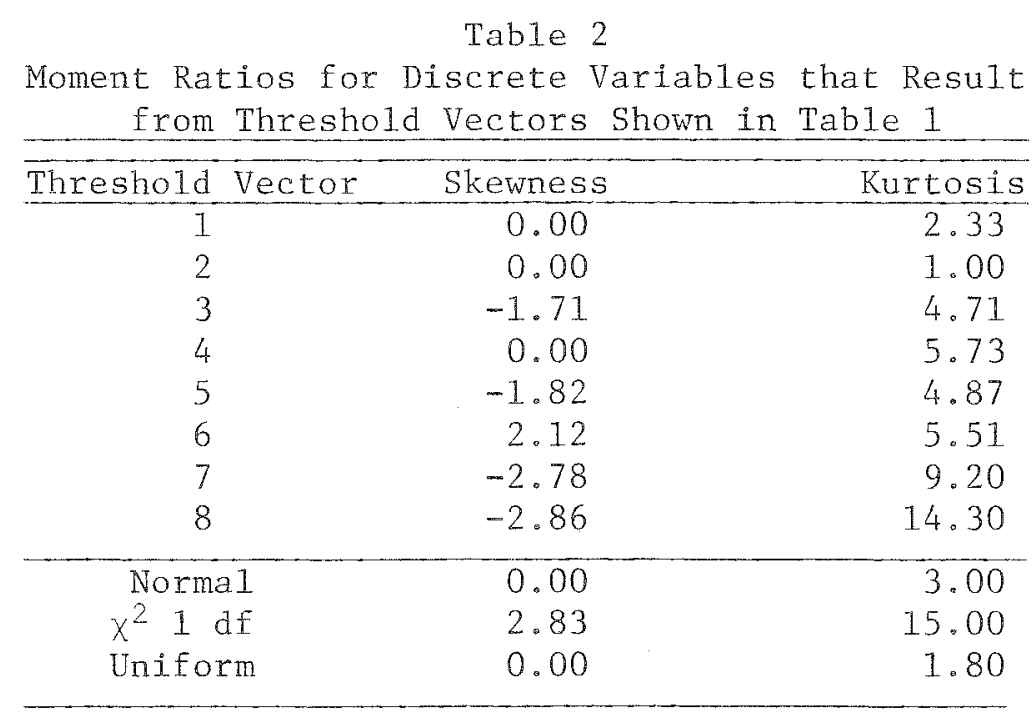

The design and results of a monte carlo study investigating the effects of categorization in multiple regression are described in the following section.

\section{Mrethod}

\section{Design of Singmalation}

Because $\mathbb{R}_{p}^{2}$ is invariant with respect to any nonsingular linear transformation of the independent variables, any independent variable covariance matrix and vector of independent variable-dependent variable covariances that yield $R_{p}^{2}$ as the squared population multiple correlation may be chosen. One particularly convenient form results from (1) choosing the independent variable covariance matrix $\Sigma_{x x}$ to be an identity matrix; (2) setting all elements in the vector $\sigma_{x v}$ of independent variable-dependent variable covariances equal to zero except for the first element, which is set equal to to $R_{p}$; and (3) choosing a dependent variable variance $\sigma_{v}^{2}$ of unity. 
The density function of sample squared multiple correlations that would result from predicting $Y$ (if it were observable) from $k$ independent variables in samples of size $N$ is

$$
\begin{aligned}
f_{S}{ }_{S}\left(p^{2}\right)=\frac{\left(1-R^{2}\right)^{\frac{1}{2}(N-1)}\left(p^{2}\right)^{\frac{1}{2}(k-2)}\left(1-p^{2}\right)^{\frac{1}{2}(N-k-3)}}{B\left(\frac{1}{2} k, \frac{1}{2}(N-k-1)\right)} \\
\quad x H\left(\frac{1}{2}(N-I), \frac{1}{2}(N-1), \frac{k}{2}, p^{2} R_{p}^{2}\right),
\end{aligned}
$$

where $\mathbb{B}$ is the beta function and $\mathbb{H}$ is the hypergeometric function (Kendall \& Stuart, 1979). From Equation 10 , it is clear that the particular forms of $\Sigma_{x x}$ and $o_{x u}$ selected do not affect the sampling distribution of $R_{s^{\circ}}^{2} \mathbb{I n}$ addition, Drasgow et al. (1979) have shown that the sampling distribution of $R_{c v}^{2}$ is also unaffected by nonsingular linear transformations. Thus, the orthogonal forms of $\Sigma_{x x}$ and $\sigma_{x v}$ can be used without loss of generality.

The "rectangle-tooth-tail" (Marsaglia, Ananthanarayanan, \& Paul, 1976) method was used to generate pseudorandom normal numbers. The uniform numbers used in this procedure were generated by combining a multiplicative congruential generator and a shift register generator. To create data for a hypothetical individual on $k$ predictor variables and $\gamma, k+1$ pseudorandom independent, standardized normal numbers were sampled. The first $k$ numbers were taken as the scores on the independent variables and $Y$ was obtained by

$$
Y=Z_{\mathbb{1}} R_{p}+Z_{k+1}\left(I-R_{p}{ }^{2}\right)^{\frac{1}{2}} \text {, }
$$

where $Z_{i}$ is the $i^{\text {th }}$ pseudorandom number sampled. This procedure yields samples from a $k+1$ dimensional multivariate normal distribution, where the independent variable covariance matrix is an identity matrix, $Y$ has unit variance, and all independent variables are independent of $\gamma$ except for $X_{1}$, which has a covariance (and correlation) of $R_{p}$ with $Y$.

Each threshold vector in Table $\mathbb{1}$ was then applied to $Y$, resulting in eight "observed" dependent variables. After $N$ individuals were simulated, sample least squares regression equation and sample squared multiple correlations were computed for $\gamma$ and each of the eight categorized variables. Each $\mathbb{R}_{s}^{2}$ was then used to estimate $\mathbb{R}_{p}^{2}$ using Equations 1 and 3 . The cross-validity of each set of sample regression weights, $\hat{b}$, was computed by

$$
R_{c V}=\frac{\hat{b}^{\prime} \sigma_{x y}}{\left(\hat{b}^{\prime} \hat{b}\right)^{\frac{1}{2}} \sigma_{y}}
$$

where $\sigma_{x y}$ is the vector of population independent variable-discrete dependent variable covariances and $\sigma_{y}$ is the standard deviation of the discrete dependent variable. It is obvious that the independent variable-dependent variable covariances are zero for all but $X_{1}$, regardless of the particular threshold vector. The covariance between $X_{1}$ and a dependent variable after $Y$ has been categorized is $q r \sigma_{y}$, where $q$ has been calculated by Equation 8 . Because $\mathbb{R}_{c v}$ can be negative (and this would be important, albeit unhappy, information to the applied researcher), the squared cross-validity was given the sign of $R_{c v}$. Finally, $R_{c v}^{2}$ was estimated by Equation 4 for each of the nine sample regression equations, using the estimators of $R_{p}^{2}$ and $R_{p}^{4}$ suggested by Browne (1975).

To summarize briefly, samples of size $N$ were drawn from $a k+1$ dimensional multivariate normal distribution. The first $k$ variables were taken as the independent variables; the $(k+1)^{\text {th }}$ variable 
was constructed to be a continuous dependent variable, which would not be observed in practice. Eight threshold vectors were applied to the continuous criterion to form categorical variables. Least squares regressions were computed for each of the nine dependent variables, and the $R_{s}^{2}$ associated with each equation was computed. Two estimates of $R_{p}^{2}, R_{c v}^{2}$ and an estimate of $R_{c v}^{2}$ were computed for each equation. Thus, a total of 45 squared correlations (five squared correlations for each of the nine criteria) was obtained for each sample.

\section{Sirmedilation Parameters}

After weighing computer costs against simulating a wide variety of situations, it was decided to generate samples of size $N=30,60$, and 120 for populations with $k=4$ and 12 independent variables. Values of $R_{p}^{2}$ for the continuous dependent variable were chosen to be $.150, .236$, and .370 for two reasons. First, they span much of the range of population squared multiple correlations that underlie data sets in psychological research. The second reason can be seen in Table 3: Note that when a threshold vector for which $q=.798$ is applied to a continuous dependent variable for which $R_{p}^{2}=.370$, the categorized dependent variable has $\mathbb{R}_{p}^{2}=.236$. Thus, the sampling distribution of $R_{c v}^{2}$ and $R_{s}^{2}$ for a categorized dependent variable with $R_{p}^{2}=.236$ can be compared to their counterparts for the continuous dependent variable with $R_{p}^{2}=.236$. This comparison is interesting because previous research (Drasgow et al., 1979) has shown that Equations 4 and 1 produce accurate estimates of $R_{c v}^{2}$ and $R_{p}^{2}$, respectively, when the variables follow a multivariate normal distribution. Similar comparisons can be made when threshold vectors for which $q=.798$ are applied to the continuous dependent variable for which $\mathbb{R}_{p}^{2}=.236$, and when threshold vectors with $q=.637$ are applied to the continuous dependent variable with $\mathbb{R}_{p}^{2}=.370$ 。

Table 3

Population Squared Multiple Correlations for Continuous and Categorical Criteria

\begin{tabular}{|c|c|c|}
\hline $\mathrm{R}_{\mathrm{p}}^{2}$ for & $\mathrm{R}_{\mathrm{p}}^{2}$ for Categorica & Criteria With \\
\hline Continuous Criterion & $q^{2}=.798^{2}$ & $q^{2}=.637^{2}$ \\
\hline .370 & .236 & .150 \\
\hline .236 & .150 & .096 \\
\hline .150 & .096 & .061 \\
\hline
\end{tabular}

\section{Resulits}

\section{Sampling Distribution of $R_{s}^{2}$}

The sampling distribution of $R_{s}^{2}$ was first investigated for the continuous dependent variable in all 18 combinations of $N, k$, and $R_{p}^{2}$ to assess the adequacy of the random number generator. Gurland (1968) obtained a closed expression for the cumulative distribution function (cdf) of $R_{s}^{2}$ under the multivariate normal assumptions. It is

$$
F_{R_{S}^{2}}\left(p^{2}\right)=\sum_{j=0}^{m} b_{j} F_{k+2 j, 2 m}\left|\frac{\left(1-R_{p}{ }^{2}\right) p^{2}}{1-p^{2}}\right|
$$


where

$$
\mathrm{m}=\frac{\mathrm{N}-\mathrm{k}-\mathrm{I}}{2}
$$

$b_{j}$ is the binomial probability

$$
b_{j}=\left(\begin{array}{l}
m \\
j
\end{array}\right)\left(R_{p}^{2}\right)^{j}\left(1-R_{p}^{2}\right)^{m-j},
$$

and $F_{v, u}$ is the cumulative distribution function of a ratio $\chi_{u}^{2} / \chi_{u}^{2}$ of independent chi-square random variables with degrees of freedom $v$ and $u$, respectively (Gurland, 1968, p. 279). Unfortunately, Gurland's form in Equation 13 is appropriate only if $N-k$ is an odd number. In the present research $N-k$ was even for all combinations of $N$ and $k$. Although Gurland derived forms for the cdf when $(N-k)$ is even, these expressions involve infinite sums. Consequently, the cdfs for $(N-k)$ even were approximated using Equation 13 by averaging cdfs obtained from Equation 13 using $(N-1)$ and $(N+1)$. From other research (not presented here) it appears that the approximate cdfs have maximum errors of less than .002 for $k=4$ and .008 for $k=12$ within the scope of the present simulation parameters.

The sample distribution function of $\mathbb{R}_{s}^{2}$ was then compared to the (approximate) theoretic distribution function for each combination of $N, k$, and $R_{p}^{2}$ for the continuous dependent variable. Kolmogorov-Smirnov significance tests were performed to assess the differences between sample and theoretic functions. Table 4 summarizes the findings. Note that for the continuous dependent variable, the actual Type 1 error rate is quite near the nominal alpha level: .055 when $\alpha=.05$, .33 when $\alpha=.25$, and .56 when $\alpha=.50$. These results provide reasonably strong support for the randomness of the pseudorandom number generator and the accuracy of the approximate cdf."

The Kolmogorov-Smirnov test was then utilized to compare the sampling distribution of each categorized dependent variable to the theoretic cdf function in Equation 13. In each test the population squared multiple correlation of the categorized dependent variable was the product of the squared attenuation factor $\left(q^{2}\right)$ for the corresponding threshold vector and the $\mathbb{R}_{p}^{2}$ of the underlying continuous variable. The results of these tests are also presented in Table 4. Again, the actual rejection rate is quite close to the nominal alpha level. ${ }^{2}$ Perhaps most interesting is the actual rejection rate when $\alpha=.50$. Here the average number of rejections is 9.5 , which is quite close to the expected number of 9.0. In sum, it appears that categorization had very little effect upon the sampling distribution of $\mathbb{R}_{s}^{2}$, except for the effects of changing the value of $\mathbb{R}_{\nu}^{2}$ as specified by Equation 7.

\section{Estimation of $R_{p}^{2}$}

Due to the findings concerning the sampling distribution of $\mathbb{R}_{s}^{2}$ described in the previous section, it is apparent that Equations 1 and 3 should estimate $\mathbb{R}_{p}^{2}$ reasonably well for categorical dependent

\footnotetext{
It does not appear possible to compute the power of the Kolmogorov-Smirnov test. Kendall and Stuart (1979, pp. 483-484) provide approximate lower bounds for the power of the test. For example, if the largest difference between true and hypothesized cdfs is .1 , if this difference occurs near the median of the two distributions, and if $\alpha=.05$, then a lower bound to the power is approximately 24 . Hence, it would be expected that the null hypothesis would be rejected far more frequentily than once in 18 tests.

IIt should be noted that significance tests within a particular row of Table 4 are not independent. Although the threshold vectors are different, all were applied to the same continuous dependent variable. Within a given column of Table 4 , however, the significance tests are independent.
} 


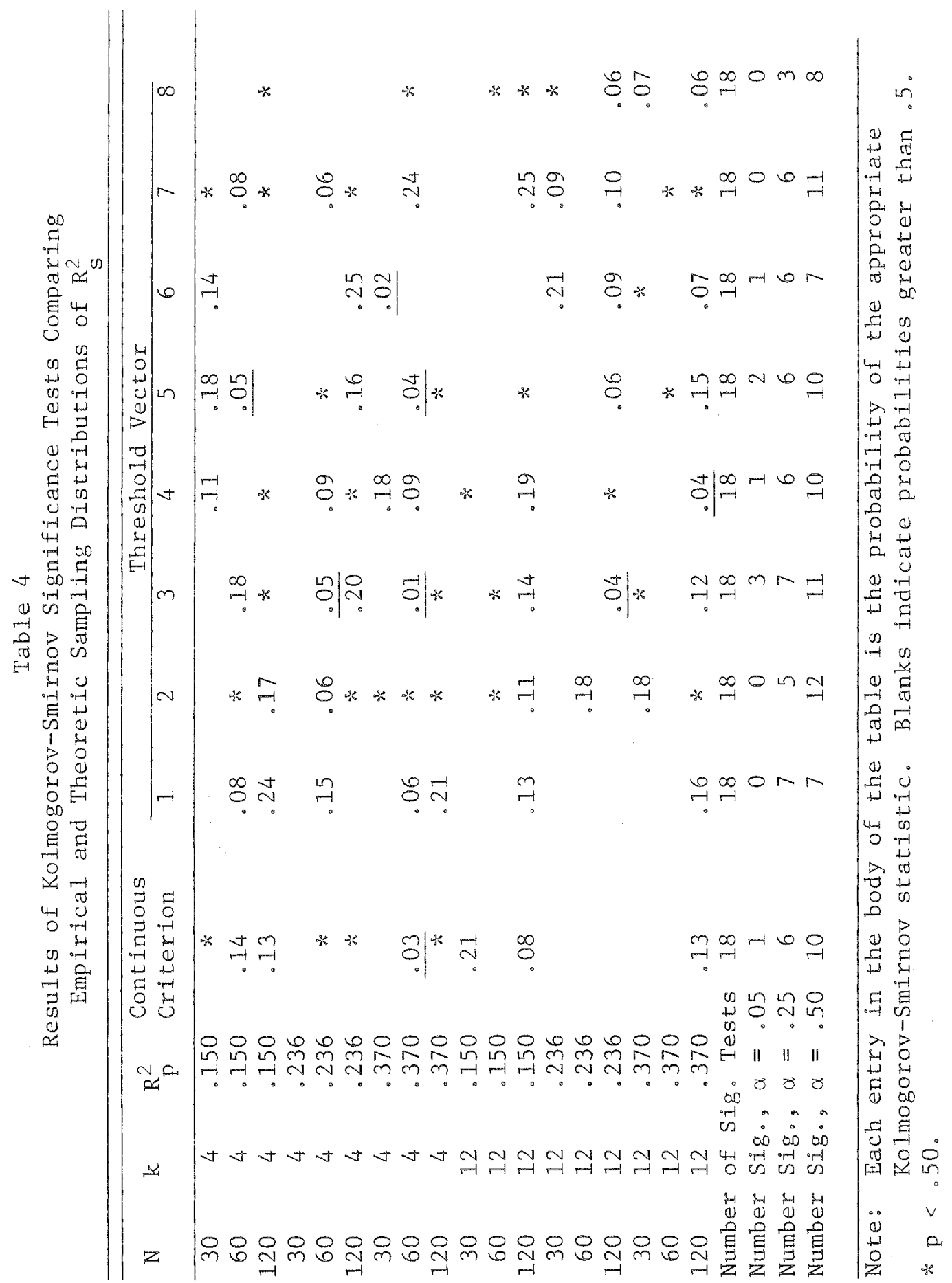

Downloaded from the Digital Conservancy at the University of Minnesota, http://purl.umn.edu/93227. May be reproduced with no cost by students and faculty for academic use. Non-academic reproduction requires payment of royalties through the Copyright Clearance Center, http://www.copyright.com/ 
variables despite the non-normality of the dependent variables. Tables 5 and 6 present mean differences between $\mathbb{R}_{p}^{2}$ and estimates of $\mathbb{R}_{p}^{2}$ obtained from Equations 1 and 3 . In Tables 5 and 6 , the columns headed by $q=1.000$ contain mean differences based upon 100 independent replications. The columns headed $q=.798$ contain entries that are based upon 400 estimates of $R_{p}^{2}$. These 400 estimates are composed of the four estimates of $\mathbb{R}_{p}^{2}$ resulting from threshold vectors $2,3,4$, and 5 across each of 100 replications. The mean differences for these threshold vectors were averaged to conserve space. Note that because the threshold vectors were applied to the same continuous dependent variable, the four estimates of $R_{p}^{2}$ are not statistically independent. Consequently, the standard errors of the mean differences presented in the columns headed $q=.798$ are not substantially smaller than the standard errors of the mean differences presented in the columns headed $q=1.000$. Mean differences for threshold vectors 6,7 , and 8 were averaged for similar reasons.

Table 7 presents the root mean squared error $(R M S E)$ of estimate of $R_{p}^{2}$ for the Wherry estimator applied to the continuous dependent variable across the 100 replications,

$$
\operatorname{RMSE}=\left\{\frac{1}{100} \sum_{i=1}^{100}\left(\mathrm{R}_{\mathrm{p}}^{2}-\hat{\mathrm{R}}_{\mathrm{p}_{i}{ }^{2}}\right)^{2 / 2} .\right.
$$

Table 7 also presents the square roots of the average $M S E$ for threshold vectors with the same value of $q$.

It is obvious that $R M S E$ is affected by the degree to which the mean estimate of $\mathbb{R}_{p}^{2}$ differs from $\mathbb{R}_{p}^{2}$. However, these differences were so small in the present study that the $R M S E$ is virtually identical

\begin{tabular}{|c|c|c|c|c|c|c|c|}
\hline \multirow{2}{*}{$\mathrm{R}_{\mathrm{p}}^{2}$} & & \multicolumn{3}{|c|}{4 Predictors } & \multicolumn{3}{|c|}{12 Predictors } \\
\hline & $q$ & $=1.000$ & .798 & .637 & 1.000 & .798 & .637 \\
\hline \multicolumn{8}{|l|}{$N=30$} \\
\hline $\begin{array}{l}.150 \\
.236 \\
.370\end{array}$ & & $\begin{array}{r}.010 \\
-.009 \\
-.013\end{array}$ & $\begin{array}{l}-.010 \\
-.011\end{array}$ & -.002 & $\begin{array}{r}.019 \\
.005 \\
-.020\end{array}$ & $\begin{array}{r}.006 \\
-.022\end{array}$ & -.025 \\
\hline \multicolumn{8}{|l|}{$N=60$} \\
\hline $\begin{array}{l}.150 \\
.236 \\
.370\end{array}$ & & $\begin{array}{l}-.016 \\
-.015 \\
-.031\end{array}$ & $\begin{array}{l}-.006 \\
-.026\end{array}$ & -.012 & $\begin{array}{l}-.004 \\
-.004 \\
-.010\end{array}$ & $\begin{array}{l}-.005 \\
-.007\end{array}$ & -.008 \\
\hline $\begin{array}{c}N=120 \\
.150 \\
.236 \\
.370\end{array}$ & & $\begin{array}{r}.003 \\
-.015 \\
.006\end{array}$ & $\begin{array}{r}-.012 \\
.004\end{array}$ & -.003 & $\begin{array}{r}.006 \\
-.008 \\
.010\end{array}$ & $\begin{array}{r}-.010 \\
.005\end{array}$ & .005 \\
\hline
\end{tabular}

Downloaded from the Digital Conservancy at the University of Minnesota, http://purl.umn.edu/93227. May be reproduced with no cost by students and faculty for academic use. Non-academic reproduction requires payment of royalties through the Copyright Clearance Center, http://www.copyright.com/ 


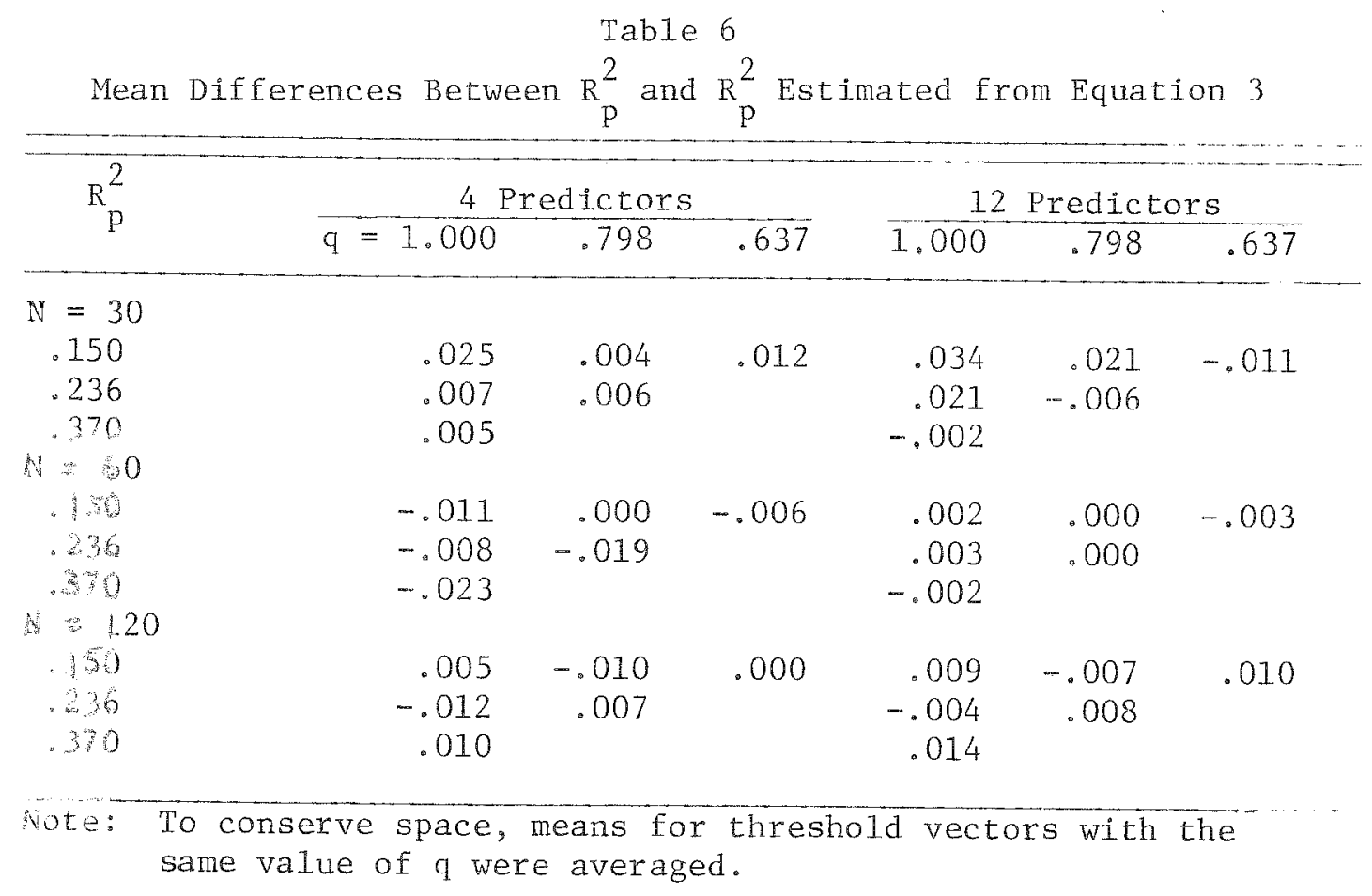

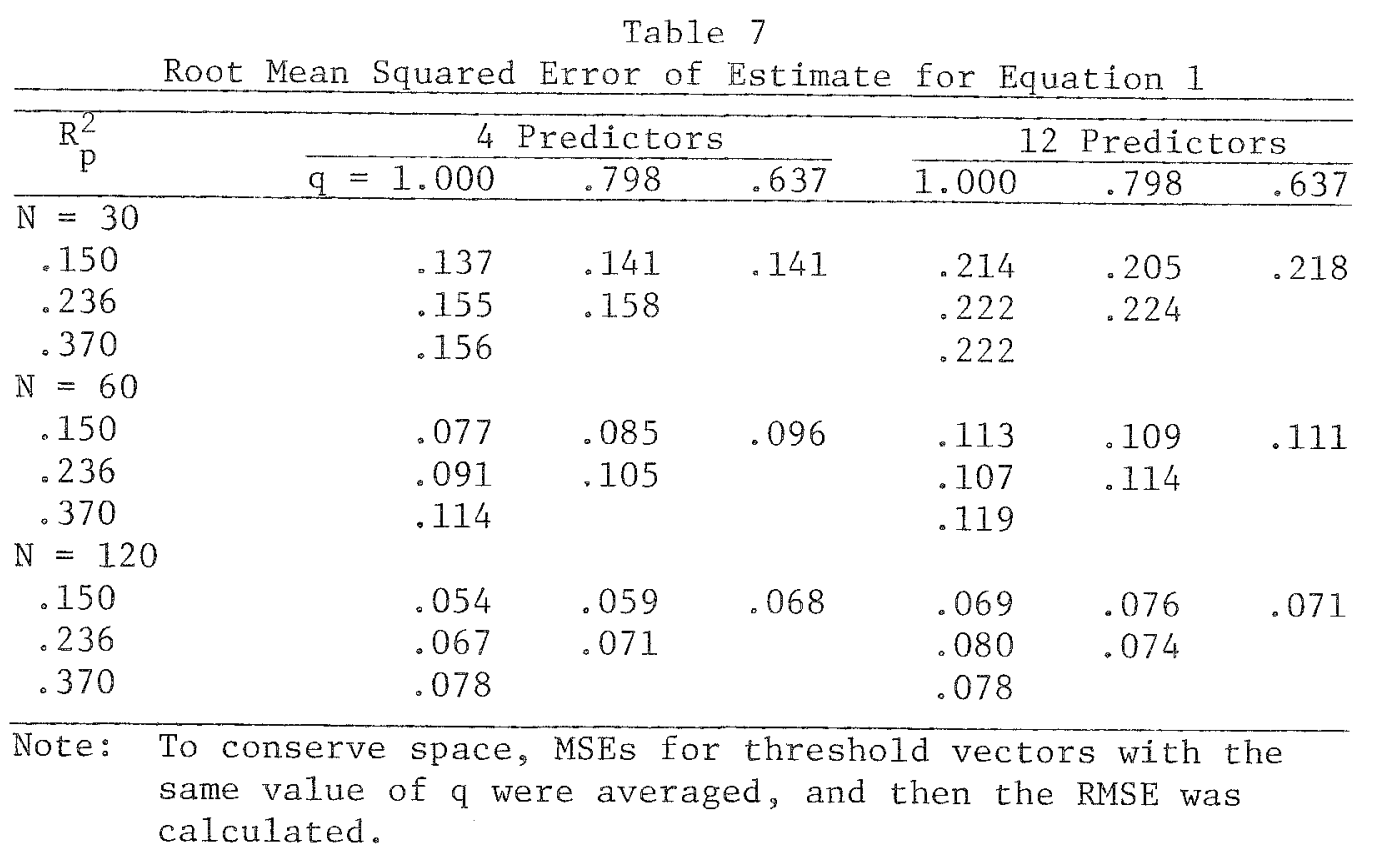

Downloaded from the Digital Conservancy at the University of Minnesota, http://purl.umn.edu/93227. May be reproduced with no cost by students and faculty for academic use. Non-academic reproduction requires payment of royalties through the Copyright Clearance Center, http://www.copyright.com/ 
to the sample standard deviations of the estimator. Consequently, the RMSE can be (1) used to understand the accuracies of the estimators and (2) divided by 10 to yield approximate standard errors for the mean differences presented in Tables 5 and 6. Finally, a table of RMSEs for the Olkin-Pratt estimator is not presented because the RMSEs for this estimator are virtually identical to RMSES seen in Table 7. The largest difference in $R M S E$ between the two estimators across all cells in Table 7 is .01 , which occurs in a sample of $N=30$. For samples of $N=120$, the RMSEs of the two estimators were generally within 001 of each other.

There are a number of interesting points that can be seen in Tables 5, 6, and 7. First, note that Equation 3 is virtually unbiased: $R_{p}^{2}$ is overestimated in 18 cells and underestimated in 14 cells. In contrast, Equation 1 has a slight tendency to underestimate $\mathbb{R}_{p}^{2}$. Here $\mathbb{R}_{p}^{2}$ is overestimated in 11 cells but underestimated in 25 cells.

An important result seen in Tables 5 and 6 is that there is no apparent tendency for Equations 1 and 3 to produce an increased bias in estimating $R_{p}^{2}$ for the categorized dependent variable. In addition, Table 7 indicates that there is very little, if any, evidence suggesting the accuracy in estimating $R_{p}^{2}$ has been reduced by categorization.

Table 7 is interesting because it provides some perspective on the sample sizes required to estimate $R_{p}^{2}$ accurately. It is clear that using 12 independent variables in a sample of 30 observations is foolish: The RMSE is enormous. The most an investigator could learn would be whether $R_{p}^{2}$ is probably "low," "moderate," or "high." The RMSE is substantially reduced for 12 predictors in samples of 120 observations. In fact, the RMSEs here are not much larger than the RMSEs for 4 predictors in samples of 120 observations. This has implications for the rule of thumb stating that the ratio of $N$ to $k$ is the critical factor in determining the stability of results in multiple regression. Apparently the rule of thumb is overly simplistic.

\section{Sarnpling $\mathbb{D}_{\text {istrilbution }}$ of $\mathbb{R}_{c v}^{2}$}

Two-sample $\mathbb{K}$ olmogorov-Smirnov significance tests were performed to assess the differences between the sampling distribution of $R_{c v}^{2}$ for continuous and categorical dependent variables. Note that $R_{p}^{2}$ for the categorical dependent variable (and not the $\mathbb{R}_{p}^{2}$ of its underlying continuous dependent variable) was used to match a categorical dependent variable with a continuous dependent variable. Table 8 presents the results of these significance tests.

From Table 8 it is clear that the sampling distributions of $R_{c v}^{2}$ for continuous and categorical dependent variables are relatively similar. For example, the actual rejection rate is quite close to the nominal rate when $a=.05$. For $\alpha=.25$, threshold vector 2 produces five rejections; however, the other threshold vectors yield frequency distributions that are significantly different from the distributions for the continuous criterion about as often as expected. Finally, when $\alpha=.50$, four of the seven threshold vectors produce one more null hypothesis rejection than expected. In sum, Table 8 does not provide compelling evidence that categorization affects the sampling distribution of $\mathbb{R}_{c v}^{2}$

\section{Estimation of $\mathbb{R}_{c v}^{2}$}

Because the continuous and categorical dependent variables yield apparently similar sampling distributions for $R_{s}^{2}$ and $R_{c v}^{2}$, it appears likely that the performance of Equation 4 will not suffer when it is used with a categorical dependent variable. Tables 9 and 10 confirm this conjecture: There is no evidence to suggest that Equation 4 is less accurate after categorization. Equation 4 shows very little (if any), mean bias for either the continuous or categorical variables. In addition, RMSEs for the categorical dependent variables are comparable to RMSEs for the continuous dependent variables. 
Table 8

Results of Kolmogorov-Smirnov Significance Tests Comparing Sampling Distributions of $\mathrm{R}^{2}$ for Continuous and Categorical Criteria

\begin{tabular}{|c|c|c|c|c|c|c|c|c|c|}
\hline \multirow[b]{2}{*}{$\mathrm{N}$} & \multirow[b]{2}{*}{$\mathrm{k}$} & \multirow[b]{2}{*}{$\begin{array}{r}\mathrm{R}^{2} \\
\mathrm{p}\end{array}$} & \multicolumn{7}{|c|}{ Threshold Vector } \\
\hline & & & 2 & 3 & 4 & 5 & 6 & 7 & 8 \\
\hline 30 & 4 & .150 & .08 & .21 & $*$ & .08 & .11 & .21 & .11 \\
\hline 60 & 4 & .150 & & & .11 & & & & $*$ \\
\hline 120 & 4 & .150 & & * & & $\therefore$ & & $\approx$ & \\
\hline 30 & 4 & .236 & & 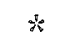 & & $\%$ & $a$ & a & a \\
\hline 60 & 4 & .236 & .06 & .08 & .03 & .08 & $\mathrm{a}$ & $\mathrm{a}$ & a \\
\hline 120 & 4 & .236 & .21 & .16 & & & a & a & $\mathrm{a}$ \\
\hline 30 & 12 & .150 & .01 & $\approx$ & $\nLeftarrow$ & & .08 & .08 & .21 \\
\hline 60 & 12 & .150 & & & * & $x$ & $*$ & & \\
\hline 120 & 12 & .150 & & $*$ & .11 & & 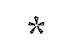 & $*$ & \\
\hline 30 & 12 & .236 & & & $\stackrel{*}{\star}$ & & a & a & a \\
\hline 60 & 12 & .236 & .21 & .21 & & .21 & $\mathrm{a}$ & a & a \\
\hline 120 & 12 & .236 & $\%$ & & *s & & a & $\mathrm{a}$ & a \\
\hline Number & of Significar & ce Tests & 12 & 12 & 12 & 12 & 6 & 6 & 6 \\
\hline Number & Significant, & $\alpha=.05$ & 1 & 0 & 1 & 0 & 0 & 0 & 0 \\
\hline Number & Significant, & $\alpha=.25$ & 5 & 4 & 3 & 3 & 2 & 2 & 2 \\
\hline Number & Significant, & $\alpha=.50$ & 6 & 8 & 8 & 6 & 4 & 4 & 3 \\
\hline
\end{tabular}

Note: The entries in the body of the table are the probabilities of the two sample Kolmogorov-Smirnov statistic. $* \mathrm{p}<.50$.

ano significance test.

\section{Discussion}

Equations 1, 3, and 4 appear to be equally accurate for discrete dependent variables and continuous normally distributed dependent variables under the conditions investigated here. The results of various Kolmogorov-Smirnov tests provide the explanation for this finding: The sampling distributions of $\mathbb{R}_{s}^{2}, \mathbb{R}_{c v}^{2}, \hat{R}_{c v}^{2}$, and the two $\mathbb{R}_{p}^{2}$ estimators were unaffected by categorization of the dependent variable beyond the attenuation of $R_{p}^{2}$. The results of this study indicate that the scope of applicability of Equations 1, 3, and 4 should be expanded to include situations where the observed dependent variable is discrete and it is reasonable to assume that there is an underlying normally distributed latent variable. Future research should be conducted to investigate the applicability of these three estimators to further violations of multivariate normality, such as categorization of the independent variables.

The robustness of most statistical methods is primarily a function of the extent to which an actual distribution differs from the assumed distribution in skewness and kurtosis. Many moment ratios of the discrete variables in Table 2 differ substantially from the corresponding normal distribution moment ratios. Differences in moment ratios larger than those seen in Table 2 could be created, but it is 
Table 9

Mean Differences Between Estimated and Actual Squared Cross-Validity

\begin{tabular}{|c|c|c|c|c|c|c|c|}
\hline \multirow{2}{*}{$\begin{array}{r}\mathrm{R}^{2} \\
\mathrm{p}\end{array}$} & \multicolumn{4}{|c|}{4 Predictors } & \multicolumn{3}{|c|}{12 Predictors } \\
\hline & $q=$ & 1.000 & .798 & .637 & 1.000 & .798 & .637 \\
\hline \multicolumn{8}{|c|}{$\mathrm{N}=30$} \\
\hline .150 & & .002 & -.015 & -.005 & .015 & .007 & .002 \\
\hline .236 & & -.002 & -.013 & & .020 & .009 & \\
\hline .370 & & -.020 & & & .020 & & \\
\hline \multicolumn{8}{|c|}{$N=60$} \\
\hline .150 & & -.020 & -.010 & -.011 & -.001 & .000 & -.002 \\
\hline .236 & & -.022 & -.024 & & .002 & -.003 & \\
\hline .370 & & -.033 & & & -.006 & & \\
\hline \multicolumn{8}{|c|}{$\mathbb{N}=120$} \\
\hline .150 & & .002 & -.012 & -.003 & .005 & -.008 & .004 \\
\hline .236 & & -.014 & .001 & & -.010 & .002 & \\
\hline .370 & & .005 & & & .007 & & \\
\hline Note: & $\begin{array}{l}\text { To cons } \\
\text { the sam } \\
\text { compute }\end{array}$ & $\begin{array}{l}\text { erve } \\
\text { e value } \\
\text { d as me }\end{array}$ & $\begin{array}{l}\text { e, mean } \\
\text { I q wer } \\
s \text { of th }\end{array}$ & $\begin{array}{l}\text { ffere } \\
\text { verag }\end{array}$ & $\begin{array}{l}\text { thres } \\
\text { ries } \\
-\mathrm{R}^{2}\end{array}$ & $\begin{array}{l}\text { d vec } \\
\text { able }\end{array}$ & $\begin{array}{l}\text { with } \\
\text { ere }\end{array}$ \\
\hline
\end{tabular}

Table 10

Root Mean Squared Errors of Estimate for Equation 4

\begin{tabular}{|c|c|c|c|c|c|c|c|}
\hline \multirow{2}{*}{$\mathrm{R}_{\mathrm{p}}^{2}$} & \multicolumn{4}{|c|}{4 Predictors } & \multicolumn{3}{|c|}{12 Predictors } \\
\hline & $q$ & $=1.000$ & .798 & .637 & 1.000 & .798 & .637 \\
\hline \multicolumn{8}{|c|}{$N=30$} \\
\hline .150 & & .132 & .129 & .125 & .124 & .120 & .115 \\
\hline .236 & & .149 & .155 & & .160 & .143 & \\
\hline .370 & & .168 & & & .164 & & \\
\hline \multicolumn{8}{|c|}{$\mathrm{N}=60$} \\
\hline .150 & & .076 & .082 & .091 & .087 & .078 & .080 \\
\hline .236 & & .095 & .102 & & .092 & .102 & \\
\hline .370 & & .119 & & & .119 & & \\
\hline \multicolumn{8}{|c|}{$\mathrm{N}=120$} \\
\hline .150 & & .055 & .056 & .066 & .060 & .064 & .062 \\
\hline .236 & & .066 & .071 & & .079 & .071 & \\
\hline .370 & & .081 & & & .080 & & \\
\hline
\end{tabular}

Note: To conserve space, MSEs for threshold vectors with the same value of $q$ were averaged, and then the RMSEs were calculated. 
not apparent that such artificial data sets would be representative of actual data sets seen in psychology. Consequently, more extreme violations of normality were not included in the present simulation.

This research has shown the importance of the categorization attenuation factor, $q$. Substantive researchers can use $q$ to assist them in deciding how many categories should be used in the measurement of a dependent variable. The categorization attenuation factor also illustrates the importance of "anchors" or labels for rating scales. Clearly, anchors should be written such that the frequencies of selecting the various categories yield a large value of $q$.

In some situations a researcher may not be able to obtain a dependent variable with a large value of $q$ (e.g., turnover data). Here an estimate of the multiple correlation obtained in predicting $Y$ may be preferred to an estimate of the multiple correlation obtained in predicting the discrete dependent variable. Work on a closely related problem has been performed by Olsson, Drasgow, and Dorans (1982), who developed a maximum likelihood estimator of the polyserial correlation. Their estimator can be used for examining the bivariate relation between an observed continuous variable and $Y$. The more difficult problem of deriving an optimal estimator for the multivariate relation between a set of continuous variables and $Y$ remains to be solved.

\section{References}

Browne, M. W. Predictive validity of a linear regres. sion equation. British Journal of Mathematical and Stutistical Psychology, 1975, 28, 79-87.

Drasgow, F., Dorans, N. I., \& Tucker, L. R. Estimators of the squared cross-validity coefficient: A monte carlo investigation. Applied Psychological Measurement. 1979, 3, 387-399.

Gurland, J. A relatively simple form of the distribution of the multiple correlation coefficient. Journal of the Royal Statistical Society, Series B, 1968, 30. 276-283.

Herzberg, P. A. The parameters of cross-validation. Psychometrika Monograph Supplement, 1969.34 (2, Pt. 2, No, 16).

Kendall, M., \& Stuart, A. The advanced theory of statistics (Vol.2, 4th ed.). New York: Macmillian, 1979.

Lord, F. M., \& Novick, M. R. Statistical theories of mental test scores. Reading MA: Addison-Wesley, 1968.

Marsaglia, G., Ananthanarayanan, K., \& Paul, N. I. Improvements on fast methods for generating normal random variables. Information Processing Letters, 1976, 5, 27-30.

Nunnally, J. Psychometric theory. New York: McGraw-Hill, 1978.
Olkin, I., \& Pratt, J. W. Unbiased estimation of certain correlation coefficients. The Annals of Mathematical Statistics, 1958, 29, 201-211.

Olsson, U., Drasgow, F., \& Dorans, N. J. The polyserial correlation. Unpublished manuscript, 1982.

Smith, P., Kendall, L., \& Hulin, C. The measurement of satisfaction in work and retirement. Chicago: Rand McNally, 1969.

Wherry, R. J. A new formula for predicting the shrinkage of the coefficient of multiple correlation. Annals of Mathematical Statistics, 1931, 2, $440-457$.

\section{ACH}

Parts of this research were originally presented at the Ledyard R. Tucker Symposium on Psychometric Theory. University of Illinois, Champaign IL. April 1980.

\section{Author's Address}

Send requests for reprints or further information to Fritz Drasgow, Department of Psychology, University of Illinois, $603 \mathrm{E}$. Daniel St., Champaign $\mathbb{I L} 61820$. 